\title{
Dysphagia in cervical dystonia patients receiving optimised botulinum toxin therapy: a single-center retrospective cohort study
}

\author{
Anna Kutschenko ${ }^{1} \cdot$ Martin Klietz $^{1} \cdot$ Lejla Paracka $^{1} \cdot$ Katja Kollewe $^{1} \cdot$ Annika Schulte-Sutum $^{1} \cdot$ Theda Janssen $^{1}$. \\ Christoph Schrader ${ }^{1} \cdot$ Florian Wegner $^{1} \cdot$ Dirk Dressler $^{1}$
}

Received: 4 May 2020 / Accepted: 17 June 2020 / Published online: 25 June 2020

(c) The Author(s) 2020

\begin{abstract}
To explore the correlations of botulinum toxin (BT) therapy with dysphagia, we wanted to study a group of cervical dystonia (CD) patients with optimised BT therapy during a prolonged period of time to record their dysphagia frequency, severity and duration, to study potential risk factors and try to avoid it by BT application with ultrasound guidance. BT therapy of $75 \mathrm{CD}$ patients (23 males, 52 females, age $60 \pm 12$ years, BT total dose $303.5 \pm 101.5 \mathrm{uMU}$ ) was retrospectively analysed for 1 year. BT therapy was optimised prior to the observation period. Dysphagia was noticed by one fifth of the patients. In those patients, it only occurred in about one third of the injection series. It was never associated with a functional deficit and lasted several days to 2 weeks. It was not related to patient age or gender, BT total dose, BT dose in the sternocleidomastoid muscle, BT dose in the sternocleidomastoid and scalenii muscles, by BT therapy with bilateral sternocleidomastoid muscle injections or BT therapy with abobotulinumtoxinA. Ultrasound guidance was not able to prevent it. Further prospective studies will be necessary to study underlying dystonia associated swallowing abnormalities as a potentially predisposing factor.
\end{abstract}

Keywords Botulinum toxin therapy $\cdot$ Cervical dystonia $\cdot$ Dysphagia $\cdot$ Adverse effects $\cdot$ Risk factors $\cdot$ Frequency

$\begin{array}{ll}\text { Abbreviations } \\ \text { ABO } & \text { AbobotulinumtoxinA } \\ \text { BT } & \text { Botulinum toxin } \\ \text { CD } & \text { Cervical dystonia } \\ \text { INCO } & \text { IncobotulinumtoxinA } \\ \text { MU } & \text { Mouse units } \\ \text { SCA } & \text { Mm. scalenii } \\ \text { SCM } & \text { M. sternocleidomastoideus } \\ \text { ONA } & \text { OnabotulinumtoxinA } \\ \text { uMu } & \text { Unified mouse units }\end{array}$

Florian Wegner and Dirk Dressler contributed equally to the manuscript.

Anna Kutschenko

Kutschenko.Anna@mh-hannover.de

1 Department of Neurology, Hannover Medical School, Carl-Neuberg-Str. 1, 30625 Hannover, Germany

\section{Introduction}

Cervical dystonia (CD) is the most common focal primary dystonia and is associated with decreased quality of life (Drexel et al. 2020). First line treatment of CD are intramuscular injections of botulinum toxin (BT). Their most common adverse effect is dysphagia. However, many aspects of this dysphagia still remain unclear including its frequency, severity, duration, reproducibility, its potential risk factors, and strategies to avoid it (Blackie and Lees 1990; Anderson et al. 1992; Comella et al. 1992, 2011; Poewe et al. 1998; Truong et al. 2010). We wanted to retrospectively study a group of CD patients with optimised BT therapy during a prolonged period of time, record the frequency, severity, duration, and reproducibility of their dysphagia, study potential risk factors, and evaluate BT application with ultrasound guidance. 


\section{Methods}

\section{Design}

This was a retrospective chart review study monitoring the occurrence of dysphagia during a period of 12 months under BT therapy. It took place at the Movement Disorders Section, Department of Neurology, Hannover Medical School from July 2018 to July 2019.

\section{Ethics}

The study was approved by the local Ethics Committee of Hannover Medical School (No. 7927_BO_K_2018). Patients gave written informed consent to the analysis and publication of their digital BT therapy data.

\section{Patients}

This study included 75 patients with CD (23 males, 52 females, age $60 \pm 12$ years) receiving regularly BT therapy of which at least one was performed under ultrasound guidance during the observation period. To reduce selection bias, only patients were included whose BT doses and other treatment parameters were previously optimised and had not been changed for at least 2 injection series prior to the observation period. Besides, it was checked that none of the patients had a history of dysphagia before BT therapy was started and that none of the patients had another diagnosis that could potentially induce dysphagia. At each injection series, patients were asked about their swallowing function. Dysphagia was defined as any reported change of swallowing in relation to the respective injection cycle.

\section{Study parameters}

Study parameters recorded included age, gender, dates of BT application, occurrence of dysphagia, injection scheme including all target muscles, target muscle doses, total BT dose, and guidance technique.

\section{BT drugs}

Patients were treated with onabotulinumtoxinA (ONA, Botox ${ }^{\circledR}$, Allergan Pharmaceuticals Ireland, Westport, Ireland), incobotulinumtoxinA (INCO, Xeomin ${ }^{\circledR}$, Merz Pharmaceuticals GmbH, Frankfurt/Main, Germany) or abobotulinumtoxinA (ABO, Dysport ${ }^{\circledR}$, Ipsen Pharma GmbH, München, Germany). INCO 100MU and ONA 100MU were reconstituted with $2.5 \mathrm{ml}$ normal saline, ABO 500MU with $5.0 \mathrm{ml}$ normal saline. Since these manufactured BT products are not formulated in the same way and their units are not determined with the same protocols, it is difficult to interconvert them other than in an arbitrary manner. Hence, for comparison of different BT drugs, doses are given as unified mouse units (uMU). Owing to the fact that most patients were treated with ONA or INCO, these doses were left unmodified (conversion ratio 1:1). Doses of ABO were divided by 2.5, a well-established conversion ratio between $\mathrm{ABO}$ and ONA as well as INCO (Wohlfarth et al. 2008; Frevert 2015; Albrecht et al. 2019). 1uMU was equal to $1 \mathrm{MU}$ of ONA, $1 \mathrm{MU}$ of INCO and 2.5MU of ABO. All BT injections were performed using a $27 \mathrm{G}$ needle of either $25 \mathrm{~mm}$ or $40 \mathrm{~mm}$ length with a $1.0 \mathrm{ml}$ syringe.

\section{Injectors}

All injectors performing the BT therapy are experienced in BT therapy and specialised in movement disorders. They were trained to perform ultrasound guided intramuscular injections and used them for at least 6 months prior to the study.

\section{Ultrasound guidance}

Ultrasound guidance was performed with a portable ultrasonic device (MyLab25Gold, Esaote Deutschland GmbH, Köln, Germany) with a $18 \mathrm{MHz}$ probe (LA435, Esaote Deutschland $\mathrm{GmbH}$, Köln, Germany) covered by a sterile transducer cover with ultrasonic transmission gel inside. The probe was placed on the skin so that the target muscle was visible in the centre of the ultrasound screen.

\section{Anatomical guidance}

Anatomical guidance was based on palpation and anatomical landmarks.

\section{Statistics}

Data are shown as mean \pm standard deviation. To check for significance of normally distributed data with equal variances the unpaired student's $t$ test was used. Otherwise, the Wilcoxonranksum-test was used. To test the association between two different groups, the chi-square test was used. The significance level was defined as 5\% $(p<0.05)$. The statistics were performed with Microsoft Excel 2017 and MATLAB R2018a.

\section{Results}

\section{Frequency}

Fifteen patients (20\%) of the 75 patients studied experienced dysphagia during the observation period. 


\section{Reproducibility}

In the group of patients with dysphagia (63 BT injections), it occurred after $32 \pm 19 \%$ of BT applications (20 BT injections). In 12 patients dysphagia occurred only once during the observation period, in 1 patient twice and in two patients three times.

\section{Severity}

If dysphagia occurred, it was reported only as a change of the sensation of swallowing. In none of these events eating habits had to be changed or physicians had to be consulted. There was no aspiration, asphyxia or pneumonia. None of the patients stopped BT therapy because of dysphagia.

\section{Duration}

The duration of symptoms was between a few days to 2 weeks, before it spontaneously disappeared.

\section{Predictability}

Potential risk factors related to dysphagia are shown in Table 1. BT total doses in patients with dysphagia (63 BT injections) were $269.9 \pm 69.9 \mathrm{uMU}$, in patients without dysphagia (263 BT injections) 315.5 $\pm 103.4 \mathrm{uMU}$
(Wilcoxon-ranksum-test, $p<0.001$ ). BT doses in the sternocleidomastoid muscle were $37.9 \pm 16.5 \mathrm{uMU}$ in patients with dysphagia and $40.8 \pm 19.2 \mathrm{uMU}$ in patients without dysphagia (Wilcoxon-ranksum-test, $p=0.242$ ). BT doses in the sternocleidomastoid (SCM) and scalenii muscles were $35.2 \pm 15.5 \mathrm{uMU}$ in patients with dysphagia and $37.9 \pm 18.5 \mathrm{uMU}$ in patients without dysphagia (Wilcoxonranksum-test, $p=0.226$ ). Other factors including patient age and gender and BT therapy with bilateral SCM injections were not significantly associated with the occurrence of dysphagia.

\section{Ultrasound guidance}

In patients without dysphagia, ultrasound guidance was used in 96 injections and anatomical guidance in 167 injections. In patients with dysphagia 21 injections were done with ultrasound guidance and 42 injections with anatomical guidance (Chi-square test, $p=0.638$ ). When ultrasound guidance was used in patients with dysphagia, 3 BT applications induced dysphagia and 18 applications did not evoke it. When anatomical guidance was used in patients with dysphagia, this side effect resulted after 17 applications and dysphagia did not occur in 25 applications (Chi-square test, $p=0.035$ ).

Table 1 Potential risk factors for dysphagia in botulinum toxin therapy

\begin{tabular}{|c|c|c|c|}
\hline Factor & Patients with dysphagia & $\begin{array}{l}\text { Patients without dys- } \\
\text { phagia }\end{array}$ & Significance \\
\hline Female sex $[n]$ & 13 & 39 & \multirow{2}{*}{$\begin{array}{l}\text { Chi-square test } \\
p=0.104\end{array}$} \\
\hline Male sex $[n]$ & 2 & 21 & \\
\hline Patient age [years] & $56.4 \pm 11.5$ & $61 \pm 12$ & $\begin{array}{l}\text { Unpaired } T \text {-test } \\
p=0.191\end{array}$ \\
\hline Total number of injection series $[n]$ & 63 & 263 & N/A \\
\hline Injection series per patient and year $[n]$ & $4.1 \pm 0.8$ & $4.1 \pm 0.8$ & $\begin{array}{l}\text { Unpaired } T \text {-test } \\
p=0.979\end{array}$ \\
\hline BT total dose [uMU] & $269.9 \pm 69.9$ & $315.5 \pm 103.4$ & $\begin{array}{l}\text { Wilcoxon-ranksum-test } \\
p<0.001\end{array}$ \\
\hline BT dose in SCM [uMU] & $37.9 \pm 16.5$ & $40.8 \pm 19.2$ & $\begin{array}{l}\text { Wilcoxon-ranksum-test } \\
p=0.242\end{array}$ \\
\hline $\mathrm{BT}$ dose in SCM+SCA [uMU] & $35.2 \pm 15.5$ & $37.9 \pm 18.5$ & $\begin{array}{l}\text { Wilcoxon-ranksum-test } \\
p=0.226\end{array}$ \\
\hline BT therapy with unilateral SCM injections $[n]$ & 41 & 159 & \multirow{2}{*}{$\begin{array}{l}\text { Chi-square test } \\
p=0.498\end{array}$} \\
\hline BT therapy with bilateral SCM injections $[n]$ & 22 & 104 & \\
\hline BT therapy with ultrasound guidance $[n]$ & 21 & 96 & \multirow{2}{*}{$\begin{array}{l}\text { Chi-square test } \\
p=0.638\end{array}$} \\
\hline BT therapy with anatomical guidance $[n]$ & 42 & 167 & \\
\hline BT therapy with ONA + INCO $[n]$ & 55 & 228 & \multirow{2}{*}{$\begin{array}{l}\text { Chi-square test } \\
p=0.898\end{array}$} \\
\hline BT therapy with $\mathrm{ABO}[n]$ & 8 & 35 & \\
\hline
\end{tabular}

ABO abobotulinumtoxinA, INCO incobotulinumtoxinA, ONA onabotulinumtoxinA, BT botulinum toxin, SCA scalenii muscles, SCM sternocleidomastoid muscle 


\section{Discussion}

\section{Frequency}

One fifth of our patients reported dysphagia. For none of them dysphagia was more than a mere observation without any functional impairment. In the literature, frequency data on dysphagia are contradictory and range from 6 to $44 \%$ of injection series (Blackie and Lees 1990; Anderson et al. 1992; Comella et al. 1992, 2011; Poewe et al. 1998; Truong et al. 2010). This may be caused by pre-described treatment protocols not allowing adequate individualisation and optimisation of the treatment schemes during the first injection series. This is typically the case in registration studies with pre-described fixed injection schemes. We are here, for the first time, presenting real-life frequency data as our patients were studied after at least one year of prior treatment optimisation. We believe that only these data may give patients and injectors an adequate and realistic understanding of the dysphagia risk in BT therapy.

\section{Reproducibility}

Another important novel finding of our study was that in those patients experiencing dysphagia this phenomenon did not occur regularly, but only in about one third of the injection series applied, further reducing the relevance of this phenomenon.

\section{Severity}

Contradictory data in the literature may also be caused by different definitions of dysphagia ranging from dysphagia as an observation (producing high dysphagia frequencies) to dysphagia resulting in functional deficits (with low dysphagia frequencies). For the sake of maximal sensitivity, we used a definition of dysphagia as an observation, thus, including even reports of the mildest forms of dysphagia, well aware that their relevance for the patient's decision to undergo BT therapy may be limited.

\section{Duration}

Whenever dysphagia occurred in our patient series its duration was limited from several days to 2 weeks maximum.

\section{Predictability}

In our study, we evaluated potential risk factors for the occurrence of dysphagia. Due to the proximity of the sternocleidomastoid and scalenii muscles to the pharyngeal muscles, the injection of these muscles is discussed in the literature as a potential risk factor for BT associated dysphagia (Anderson et al. 1992; Comella et al. 1992).That is why we included in our evaluation not only patient age and gender, BT total dose, BT therapy with $\mathrm{ABO}$ and $\mathrm{BT}$ therapy with anatomical guidance but also BT dose in the sternocleidomastoid muscle, BT dose in the sternocleidomastoid and scalenii muscles, and BT therapy with bilateral sternocleidomastoid muscle injections. None of these factors, however, was related to the dysphagia risk. The trend towards reduced BT total doses, decreased BT doses in the sternocleidomastoid muscle and the sternocleidomastoid and scalenii muscles may have been caused by dose reductions during the dose optimisation phase.

\section{Ultrasound guidance}

Our data indicate that ultrasound guidance does not reduce the overall dysphagia risk, whilst in some patients with dysphagia it may reduce it. Although ultrasound guidance is helpful for BT placement in children and in forearm muscles of adults (especially in writer's cramp where dystonia involvement may be highly selective) (Walter and Dressler 2014), BT placement in CD seems to be equally safe when based on anatomical landmarks and palpation.

\section{Limitations of the study}

The patients' data were only retrospectively analyzed and the study was not randomized. Furthermore, the occurrence of side effects was documented during the patients' anamnesis interviews before the next BT injection when these symptoms were no longer present. An objective clinical evaluation of side effects few weeks after the BT injections was not carried out. Besides, the group of patients with dysphagia was markedly smaller than the group of patients without dysphagia as well as the group of $\mathrm{ABO}$ treated patients was smaller than the group of INCO or ONA treated patients so that the results have to be interpreted cautiously.

\section{Conclusions}

In $\mathrm{CD}$ patients with optimised BT therapy dysphagia may be noticed by one fifth of the patients in about one third of the injection series. It is not associated with a functional deficit and may last several days to 2 weeks. It was not related by patient age or gender, BT total dose, BT dose in the sternocleidomastoid muscle, BT dose in the sternocleidomastoid and scalenii muscles, by BT therapy with bilateral sternocleidomastoid muscle injections, or BT therapy with ABO. Ultrasound guidance is not able to prevent it. Further studies 
will be necessary to study underlying dystonia associated swallowing abnormalities as a potentially predisposing factor.

Acknowledgements Open Access funding provided by Projekt DEAL.

Author contributions AK designed and conceptualized the study. AK, MK, LP, KK, ASS, TJ, and CS performed the data collection. AK, FW, and DD analysed the data. AK wrote the first draft of this manuscript. MK, FW, and DD made substantial contributions to the writing and language editing of the final draft and enhanced the quality of the manuscript by revising it critically for important intellectual content. DD finalized the manuscript for intellectual content. All authors read and approved the final manuscript.

Funding This study did not receive any specific funding.

Data availability All data are available within the text of the article. Further anonymised data could be made available to qualified investigators upon reasonable request.

\section{Compliance with ethical standards}

Conflict of interest DD received honoraria for services provided to Allergan, Ipsen, Merz, Lanzhou Institute of Biological Products, MedyTox, Revance, Desitin, Syntaxin, Abbvie, Medtronic, St Jude, Boston Scientific, Almirall, Bayer, Sun, Teva, UCB, IAB-Interdisciplinary Working Group for Movement Disorders. He is shareholder of Allergan and holds patents on botulinum toxin and botulinum toxin therapy. He provides professional consulting services to pharmaceutical companies and professional investment institutions on all aspects of botulinum toxin drugs. MK received honoraria for services provided to Novartis and has received research support for the German "Arbeitskreis Botulinumtoxin". KK received travel grants and/or honoraria for lectures from Allergan, Biogen, Ipsen, Lilly, Merz, Novartis, Teva. AK, LP, ASS, TJ, FW have no conflict of interest to report.

Ethical approval The study was approved by the local Ethics Committee of Hannover Medical School. All the procedures being performed were part of the routine care.

Consent to participate Patients gave written informed consent to the restrospective analysis of their digital BT therapy data.

Consent for publication Patients gave written informed consent for the publication.

Open Access This article is licensed under a Creative Commons Attribution 4.0 International License, which permits use, sharing, adaptation, distribution and reproduction in any medium or format, as long as you give appropriate credit to the original author(s) and the source, provide a link to the Creative Commons licence, and indicate if changes were made. The images or other third party material in this article are included in the article's Creative Commons licence, unless indicated otherwise in a credit line to the material. If material is not included in the article's Creative Commons licence and your intended use is not permitted by statutory regulation or exceeds the permitted use, you will need to obtain permission directly from the copyright holder. To view a copy of this licence, visit http://creativecommons.org/licenses/by/4.0/.

\section{References}

Albrecht P, Jansen A, Lee JI, Moll M, Ringelstein M, Rosenthal D, Bigalke H, Aktas O, Hartung HP, Hefter H (2019) High prevalence of neutralizing antibodies after long-term botulinum neurotoxin therapy. Neurology 92(1):e48-e54

Anderson TJ, Rivest J, Stell R, Steiger MJ, Cohen H, Thompson PD, Mardsden CD (1992) Botulinum toxin treatment of spasmodic torticollis. J R Soc Med 85(9):524-529

Blackie JD, Lees AJ (1990) Botulinum toxin treatment in spasmodic torticollis. J Neurol Neurosurg Psychiatry 53(8):640-643

Comella CL, Tanner CM, DeFoor-Hill L, Smith C (1992) Dysphagia after botulinum toxin injections for spasmodic torticollis: clinical and radiolgic findings. Neurology 42(7):1307-13310

Comella CL, Jankovic J, Truong DD, Hanschmann A, Grafe S, U.S. XEOMIN Cervical Dystonia Study Group (2011) Efficacy and safety of incobotulinumtoxinA (NT 201, XEOMIN®, botulinum neurotoxin type A, without accessory proteins) in patients with cervical dystonia. J Neurol Sci 308(1-2):103-109

Drexel SC, Klietz M, Kollewe K, Paracka L, Kutschenko A, Kopp B, Lange F, Wegner F, Dressler D (2020) Caregiver burden and health-related quality of life in idiopathic dystonia patients under botulinum toxin treatment: a cross-sectional study. J Neural Transm 127(1):61-70

Frevert J (2015) Pharmaceutical, biological, and clinical properties of botulinum neurotoxin type A products. Drugs R D 15(1):1-9

Poewe W, Deuschl G, Nebe A, Feifel E, Wissel J, Benecke R, Kessler KR, Ceballos-Baumann AO, Ohly A, Oertel W, Künig G (1998) What is the optimal dose of botulinum toxin A in the treatment of cervical dystonia? Results of a double bling, placebo controlled, dose ranging study using Dysport. German Dystonia Study Group. J Neurol Neurosurg Psychiatry 64(1):13-17

Truong D, Brdodsky M, Lew M, Brashear A, Jankovic J, Molho E, Orlova O, Timerbaeva S, Global Dysport Cervcal Dystonia Study Group (2010) Long-term efficacy and safety of botulinum toxin type A (Dysport) in cervical dystonia. Parkinsonism Relat Disord 16(5):316-323

Walter U, Dressler D (2014) Ultrasound-guided botulinum toxin injections in neurology: technique, indications and future perspectives. Expert Rev Neurother 14:923-936

Wohlfarth K, Schwandt I, Wegner F, Jürgens T, Gelbrich G, Wagner A, Bogdahn U, Schulte-Mattler W (2008) Biological activity of two botulinum toxin type A complexes (Dysport and Botox) in volunteers: a double-blind, randomized, dose-ranging study. J Neurol 255(12):1932-1939

Publisher's Note Springer Nature remains neutral with regard to jurisdictional claims in published maps and institutional affiliations. 